\title{
Survey on the Research Challenges of Radio Resource Management in LTE-A and the Current Proposed Solutions for these Challenges
}

\author{
Huthaifa Al-Jaradat, Kumbesan Sandrasegaran \\ Faculty of Engineering and Information Technology \\ University of Technology Sydney \\ h.aljeradat@gmail.com \\ kumbes@eng.uts.edu.au
}

\begin{abstract}
Long Term Evolution-Advanced (LTE-Advanced) has been recently submitted by the $3^{\text {rd }}$ Generation Partnership Project (3GPP) to the International Telecommunication Union (ITU) as one of the candidates 4G technologies. LTE-Advanced is expected to outperform its predecessor (i.e. LTE) by providing data rate up to $1 \mathrm{Gbps}$ and $500 \mathrm{Mbps}$ in the downlink and uplink directions, respectively, also by supporting higher speed mobility (i.e. $500 \mathrm{~km} / \mathrm{h}$ ). In order to allow such advances in the performance, Radio Resource Management (RRM) must be effectively utilized. This paper studies the technical challenges associated with some of the RRM tasks (including Packet scheduling, interference management and handover control), in addition it presents from the open literature some of the proposed solutions to these technical challenges.
\end{abstract}

\section{Council for Innovative Research}

Peer Review Research Publishing System

Journal: INTERNATIONAL JOURNAL OF COMPUTERS \& TECHNOLOGY

Vol 7, No 3

editor@cirworld.com

www.cirworld.com, member.cirworld.com 


\section{Introduction}

In order to meet the requirements of the International Mobile Telecommunication Advanced (IMT-Advanced), defined by the International Telecommunication Union Radiocommunication sector (ITU-R), 3rd Generation Partnership Project (3GPP) has proposed the LTE-Advanced, or the so-called LTE Release 10 (LTE Rel-10[1]. LTE-Advanced is expected to outperform its predecessor (i.e. LTE Rel-8), by supporting data rate up to $1 \mathrm{Gbps}$ and 500 Mbps in the downlink and uplink directions, respectively [2]. 3GPP has adopted different access technologies in the downlink and uplink directions of the LTE-Advanced. In the downlink direction, in order to support high data rate and high spectrum efficiency Orthogonal Frequency Division Multiple Access (OFDMA) is used. In the uplink direction, due to the limited power in the UE's battery Single-Carrier Frequency Division Multiple Access (SC-FDMA) is used [3]. The radio architecture of the LTE-Advanced is shown in Figure 1.

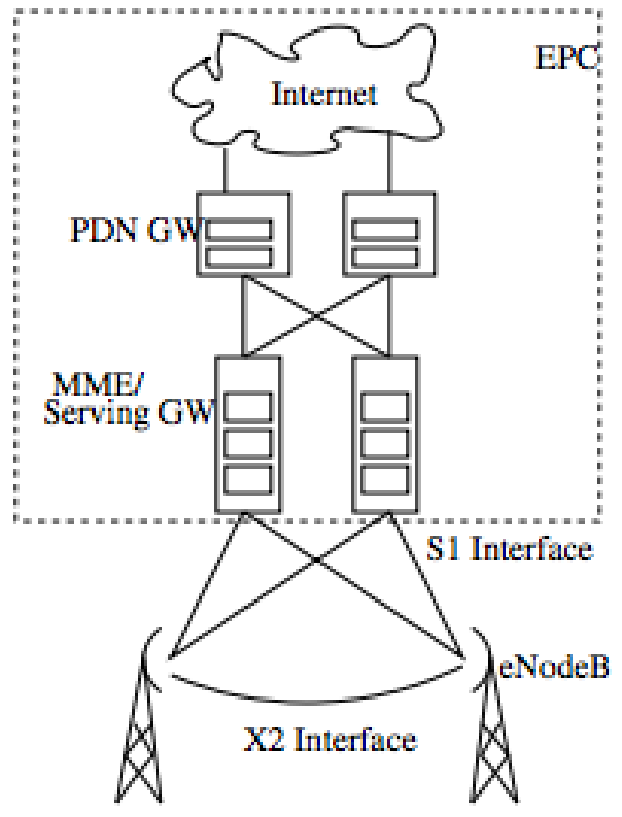

Figure 1: Network Architecture of LTE-Advanced [4]

The architecture of the LTE-A network comprises three main elements: evolved Node Base station (eNB), Mobility Management Entity (MME), and Serving Gateway (S-GW)/ Packet Data Network Gateway (PDN-GW or called P-GW). The eNB provides User Equipment (UE) with the necessary user and control plane protocols over the air interface, additionally it handles all Radio Resource Management (RRM) aspects such as packet scheduling and handover mechanism. MME is responsible for user authentication and bearer management. S-GW and P-GW are the anchor points towards E-UTRAN and packet data network, respectively [5].

In order to meet the requirements of IMT-Advanced, LTE-Advanced adopts several new advanced technologies [6]. For instance, Carrier Aggregation (CA) has been proposed to extend the transmission bandwidth from $20 \mathrm{MHz}$ up to $100 \mathrm{MHz}$, hence increasing the user's data rate. Another trend that is expected to enhance the performance of the LTE-Advanced is the Multiple Input Multiple Output (MIMO) technique. LTE-Advanced is expected to support up to $8 \times 8$ and $4 \times 4$ antenna configuration in the downlink and uplink directions respectively. Coordinated Multi-Point (CoMP) transmission/reception, where multiple eNBs are cooperated in order to enhance the performance of cell edge users, is anticipated to mitigate outages at cell edge. Finally, the Relay nodes and Heterogeneous Networks (HetNet) improve coverage and capacity in areas difficult or expensive to reach using the conventional approach.

The purpose of this paper is to study some of the technical challenges that face the radio resource management in the LTE-Advanced and some of the proposed solution to these challenges from the open literature. The rest of this paper is organized as follows: Section II gives an overview of the challenges that face packet scheduling and some of the proposed algorithms from the literature. Section III studies the technical challenges and the proposed solution for interference management, and Section IV studies the handover mechanisms in the LTE-A. Finally the paper is concluded in Section v.

\section{Packet Scheduling}

Packet Scheduling (PS) is the process of dividing and allocating resources between users who have data to transfer. PS has a vital role in determining system performance, as it responsible to allocate resources to users, both in the time and frequency domains, based on the Channel State Information (CSI) report from user in the uplink direction. The purpose of the PS algorithms is to fulfill the Quality of Service (QoS) and fairness demands of each user along with effective utilization of the available radio resources [7]. PS over the LTE-Advanced faces a challenge of supporting diverse QoS requirements, such as reduced packet delay, and Packet Drop Rate (PDR) of Real Time (RT) traffic and high throughput of Non Real Time Traffic (NRT) streaming video, while maintaining the overall system throughput and fairness among users at an acceptable level [8]. Another challenge that faces PS over LTE-Advanced is due to the use of CA. With CA, 
some users can access up to five Component Carriers (CCs), while other may access only one CC. Which gives a challenge to the packet scheduler to optimize the system performance over all CCs [9]. The adoption of CoMP scheme, where the scheduling decision for each user is taken among several eNBs, represents also new technical challenge when considering the challenges of PS over LTE-Advanced. By surveying the open literature, we provide below some of the algorithms that are proposed to deal with PS over LTE-Advanced.

\section{A. Cross-CC Packet Scheduling Algorithm}

LTE-A is designed to support a $100 \mathrm{MHZ}$ transmission bandwidth by aggregating up to five CCs. Therefore, the packet scheduler must take into account PS in multiple CCs environment [10]. In [11], the authors introduced two PS metrics; independent PS per CC and cross-CC PS, and then they proposed the use of Proportional Fair (PF) scheduler to allocate resources based on the cross-CC PS metric. The reason to choose the PF is that, the PF has a full knowledge of the frequency selective channel conditions of each user, therefore it offers frequency domain packet scheduling gain. The PF grants resources to the user who maximizes the following metric on each CC.

$K_{\mathrm{i}, j}=\arg \max \left\{M_{k_{i}, j, j}\right\}$

(1)

Where $k_{i_{i} j}$ is the selected user on the $i^{\text {th }} \mathrm{CC}$ at the $j^{\text {th }}$ Physical Resource Block (PRB). PRB is the smallest transmission unit in the downlink LTE-Advanced. PRB comprises 12 sub-carriers, note that the sub-carriers are spaced by $15 \mathrm{KHz}$ and the PRB has $180 \mathrm{KHz}$ overall bandwidth. And $M_{k_{i} i_{j} j}$ is the scheduling metric that can be calculated either independent per $\mathrm{CC}$ or cross-CC as given by (2) and (3) respectively.

- Independent PS per CC, similar to the traditional single carrier system, where the scheduling metric is calculated for individual CC without considering transmission characteristics on other CCs, it is calculated by dividing the instantaneous throughput by the average throughput as below:

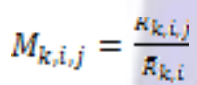

Where $R_{k_{i} j}$ is the throughput of user $k$ on the $i^{\text {th }} \mathrm{CC}$ at the $j^{\text {th }} \mathrm{PRB}$ group and $\bar{R}_{k j i}$ is the average throughput of user $k$ on the same CC in the past.

- Cross-CC PS, by considering all the statistics from all CCs, PS can achieve better resource allocation than independent PS. The scheduling metric is calculated by dividing the instantaneous throughput by the past user throughput over all aggregated CCs as below:

$$
M_{k_{i}, i j j}=\frac{k_{k, i j} j}{\sum_{i=1}^{N} R_{k i j}}
$$

B. User Group Proportional Fair (UG-PF) algorithm

The authors of [12] developed algorithm known as User Group Proportional Fair (UG-PF) for supporting PS in Carrier Aggregation (CA) mode, especially inter-band CA mode. The UG-PF algorithm divides users into different groups according to the number of CCs that users can access, and then allocates PRBs to different user groups according to the average channel access probability of these groups and number of CCs that users can be scheduled on. In UG-PF, resources are allocated to user $\vec{i}^{*}$ who belongs to user group based on Equations (4) \& (5).

$(4)$

s.t. $M=M_{k} \cup M_{k+1} \ldots \cup M_{L}=\bigcup_{j=k}^{L} M_{j}$

(5)

$$
\beta=\frac{R^{2}}{R_{j}^{2}}
$$

(6)

Where $i^{*}$ is the user who maximizes the ratio of the achievable instantaneous data rate over the average received data rate, $r_{i}(n, s)$ is the achievable instantaneous data rate of user $i$ on PRB $n$ at time slot $s, \overline{R_{i}(s)}$ is the average data rate of user $i, M$ is the user group that user $i$ belong to, $j$ is the number of CCs that user $i$ can be scheduled on, $L$ is the number of CCs which the system can aggregate, $k$ is variable denotes number of CCs, $R$ is the coverage radius of the CC and $\beta$ is weighting factor that adjusts the average channel access probability of different user groups relative to the number of CCs that they can be scheduled on.

\section{Efficient Proportional Fair (EPF) algorithm}

An algorithm known as Efficient Proportional Fair (EPF) was developed in [10] to provide support for LTE-A downlink transmissions supporting both Real Time (RT) and Non Real time (NRT) traffics simultaneously. In EPF algorithm, a classifier unit classifies the arrived packets into RT packets and NRT packets, and then delivers it into RT queue and NRT queue, respectively, based on the rule first-come-first-served. The NRT packets can be scheduled only on a part of the 
available PRBs, if there are $b_{\text {total }}$ PRBs then the number of PRBs available for NRT is given by $b_{\text {NRT }}=\eta b_{\text {total }}$, where $\eta$ is a predetermined value. For RT packets $\delta_{R T}$ is defined as the delay constraint for each RT packet and the RT packets can be scheduled on $(1-\eta) b_{\text {total }}$ PRBs. The scheduler follows the following 8 steps in the scheduling process:

Step 1: Transferring NRT packets stored in the NRT queue from previous runs (if there) to the transmission queue, notice there are only $\eta b_{\text {total }}$ PRBs available to NRT packets.

Step 2: Transferring RT packets stored in the RT queue from previous runs (if there) to the transmission queue.

Step 3: In each run, if the transmission delay of RT packets exceeds the delay constraint $\left(\delta_{R T}\right)$, drop the packet.

Step 4: Start from the unused PRB on the first CC to schedule packets in the transmission queue.

Step 5: Calculate the fairness metric, Equation (7), modified from the PF equation.

$\operatorname{tuple}\left(i^{*} j^{*} k^{*}\right)=\arg \max _{i_{v} j, k} \frac{r_{k}\left(\bar{i}_{j} j g\right)}{\overline{r_{k}}}$

Where $n_{k}\left(i_{i} j_{s} s\right)$ is the instantaneous data rate of user $k$ on the $i^{\text {th }} \mathrm{CC}$ at the $j^{\text {th }} \mathrm{PRB}$ in the current scheduling run $s$, and $\overline{\eta_{k}}$ is the average data rate of user $k$ after the end of last scheduling interval.

Step 6: Schedule user $k^{*}$, the one who maximized Equation (7), on the $j^{\text {th }}$ PRB in the $i^{\text {th }} \mathrm{CC}$. Note that, if the user's packet is larger than the size of the PRB, it will be partitioned, and the remaining parts may be transmitted in the same or next runs.

Step 7: Update users' average data rate using Equation (8).

$\overline{n_{k}}=\left(1-\frac{1}{\tau}\right) \overline{n_{k}}+\frac{1}{\tau} n_{k}\left(i_{v} j, s\right)$

Where $\tau$ is constant to average users' data rate.

Step 8: Check if there are still enough resources that can be allocated in this run. If yes, go back to step 4, otherwise, go back to step 1 and start new run.

D. Proportional Fair Scheduling for Multi-Cell Multi-User MIMO systems.

The authors of [13] proposed an algorithm known as Proportional Fair (PF) for Multi-cell Multi-User MIMO systems, for exploring multi-user diversity and combating both intra-cell and inter-cell interference in the downlink of LTE-A. The proposed algorithm can be used for scheduling users in both single cell and multi-cell Multi User MIMO (MU-MIMO) systems. For single cell MU-MIMO, if there are $N$ users in a cell, the Base Station (BS) selects $n$ users to serve in frequency resources each time slot, where $n \leq N$. The scheduler selects a group of users that maximizes $l_{n}$ in Equation (9).

$l_{n}=\sum_{k \in g_{n}} \frac{R_{k}^{n}}{T_{k}}$

Where $I_{n}$ denotes the rate of change of the utility function for the selected group of users in a single cell mode, $s_{n}$ is the subset of users that BS intends to transmit to, $R_{k}^{n}$ is the achievable rate $n$-tuple for the corresponding MU-MIMO system with precoding scheme and $T_{k}$ is the accumulated throughput of user $k$. In multi-cell MIMO system, each user has different number of serving BSs, which makes scheduling more difficult. In multi-cell MIMO system, the scheduler selects a group of users that maximize $l_{n}^{M C}$ in Equation (10).

$l_{n}^{M C}=\sum_{k \in S_{n}^{M C}} \frac{R_{k}^{M C, n}}{T_{k}}$

Where $I_{n}^{M C}$ denotes the rate of change of the utility function for the selected group of users in multi-cell mode, $S_{n}^{M C}$ is the subset of users that BSs intend to transmit to in a multi-cell mode, $R_{k}^{M C_{m} n}$ is the achievable $n$-tuple for the corresponding MU-MIMO system with precoding scheme and $T_{k}$ is the accumulated throughput of user $k$. It was shown via simulation result that the proposed algorithm improves the average cell throughput and cell edge throughput of LTE-A system to meet the IMT-A requirements.

\section{E. Similar Proportional Fairness (SPF) algorithm}

Similar to [12], the authors of [14] developed an algorithm for supporting joint scheduling crossing multiple CCs. Herein the algorithm is called Similar Proportional Fairness (SPF) algorithm. The idea behind the SPF algorithm is to calculate users' 
selection weight on each CC, as given by Equation (11), and then adopts the PF algorithm to allocate PRBs on CCs to the user $i^{*}$ who maximizes Equation (15).

$M_{\mathrm{i}}^{k}= \begin{cases}\frac{L_{\text {th }}}{L_{\mathrm{k}}^{k}} \times \frac{\bar{N}}{\operatorname{Num}_{k}} ; \quad \text { else } \\ 0 \quad \frac{B_{k}}{d_{i}}<1\end{cases}$
$l_{\text {th }}=58.83+37.6 \log \left(10 R_{k}\right)+21 \log \left(10 f_{k}\right)$

$L_{i}^{k}=58.83+37.6 \log \left(10 d_{i}\right)+21 \log \left(10 f_{k}\right)$

$\bar{N}=\frac{N w m_{n}}{n}$

$i^{*}=\arg \underset{i}{\max }\left\{M_{i, j}^{k}\right\}$

$M_{\mathrm{i}_{j} j}^{k}=M_{i}^{k} \times \frac{r_{i, j}}{\overline{\gamma_{i}}}$

Where $M_{i}^{k}$ is the i'th UE's selection weight on the $k^{\prime}$ th CC, $L_{t}$ is the threshold path loss; calculated from Equation (12), $L_{i}^{k}$ is the i'th UE's path loss on the k'th CC; calculated from Equation (13), $\bar{N}$ is the number of UEs that each CC can serve, $\mathrm{Num}_{k}$ is the number of UEs who can occupy resource of $\mathrm{CC}_{\mathrm{k}} \mathrm{n}$ is the number of aggregated CC, $R_{k}$ is $\mathrm{CC}_{\mathrm{k}}$ 's coverage radius, $d_{i}$ is the distance between $\mathrm{UE}_{i}$ and eNB, $f_{k}$ is the frequency of the $k^{\prime}$ th $\mathrm{CC}$ and $M_{i_{j}}^{k}$ is the UE $\mathrm{E}_{i}$ scheduling weight on the $j$ 'th PRB of the $\mathrm{CC}_{k}$.

F. Optimised cross-CC Proportional Fair (OCPF) algorithm

In the cross-CC PF algorithm, proposed by the authors of [11], if there are two users; one LTE and one LTE-A, having same transmission characteristics for the whole transmission period, and similar instantaneous data rate on all PRBs. Then in order to allocate radio resources to the LTE-A user, its scheduling metric must be higher than the LTE user's scheduling metric. In other words, LTE-A user's average data rate must equal or less than LTE user's average data rate, regardless the number of CCs that LTE-A user works on, otherwise, resources allocated to LTE user and his throughput becomes higher than LTE-A user's throughput. This is inadmissible, since supporting data rate higher than in LTE is an essential requirement of LTE-A. The authors of [15] proposed new scheduling metric (Equation (17)) that can be used with the PF scheduler, and they named it Optimised Cross-CC PF (OCPF), to overcome this imperfection.

$M_{k, i, j}=\frac{r_{k, i j}}{\left(\sum_{i=1}^{N} R_{k i} i j\left(N_{k}\right)\right)}$

$R_{k_{i j} i}(t)=\left(1-\frac{1}{t_{c}}\right) R_{k_{i j} i}(t-1)+\frac{1}{t_{r}} n_{k_{i} i}(t-1)$

Where $r_{k}, j$ is the instantaneous supportable data rate of user $k$ on the $I^{\text {th }}$ CC at the $j$ th PR at time slot $t$, $R_{\mathrm{k}, i}$ is the average data rate of user $k$ in the same CC in the past $t_{c}$ time slots, $N_{k}$ is the number of CCs that user $k$ uses, $N$ is the total number of CC, $i$ is a variable of the summation to compute across all CC and $n_{k i}(t-1)$ is the instantaneous supportable data rate of user $k$ on the $i^{\text {th }} \mathrm{CC}$ at time slot $(t-1)$.

\section{G. Improved Independent Scheduling Proportional Fair Algorithm}

To improve fairness and throughput of LTE users', when LTE and LTE-A users coexist together, an improved PF algorithm based on independent PS per CC is proposed by the authors of [16]. In the proposed scheme, users are divided into two groups; LTE users and their percentage to the overall users is represented by $\alpha$, and LTE-A users, also, the CCs is divided into two types; LTE-A CCs that can be accessed by LTE-A users only, and LTE CCs that can be accessed by all users, LTE CCs' percentage is represented by $\beta$. The scheduler follows the following 6 steps in the scheduling process:

Step 1: Based on the percentage of LTE users and LTE CCs', the eNB sets the values of $\alpha$ and $\beta$.

Step 2: The eNB compares $\alpha$ with $\beta$. If $\alpha<\beta$, go to step 3, otherwise, go to step 4 .

Step 3: LTE-A users can access all CCs (LTE and LTE-A CCS), and resources are allocated to user who maximizes scheduling metric given by Equation (19). While LTE users can access only LTE CCs', and resources allocated to user who maximizes scheduling metric given by Equation (20). Upon completion this step, the scheduler goes to Step 6. 


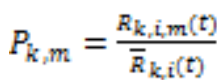

$P_{k_{i} i m}=N \beta[(1-\alpha) /(\beta-\alpha)]\left[R_{k_{i} i_{\alpha} m}(t)\right] /\left[\bar{R}_{k_{k} i}(t)\right]$

$\bar{R}_{k i j}(t+1)=\left(1-\frac{1}{T}\right) \bar{R}_{k_{i j} i}(t)+\frac{1}{T} R_{k_{i} i}(t)$

Where $P_{k_{2} m}$ is the priority of user $k$ on the $m^{\text {th }}$ PRB, $R_{k_{j}, i_{m}}(t)$ is the estimated throughput of user $k$ at the $m^{\text {th }}$ PRB group of the $i^{\text {th }} \mathrm{CC}$ at time slot $t . \bar{R}_{k i \mathrm{i}}(t)$ is the average throughput of the user $k$ on the $i^{\text {th }} \mathrm{CC}$, updated by Equation $21, P_{k_{i} \text { im }}$ is the priority of user $k$ at the $m^{\text {th }}$ PRB on the $i^{\text {th }}$ CC, $N$ is the number of CCs, $\beta$ is the percentage of LTE CCs, $\alpha$ is the percentage of LTE users, and $T$ is the average window length.

Step 4: LTE-A users only allowed accessing LTE-A CCs' and both LTE and LTE-A users scheduled on PRBs using scheduling metric given by Equation (19).

Step 5: After allocating all $m$ PRBs, update the value of the average date rate $\bar{R}_{\mathbb{k}_{i j}}(t)$ using Equation (21).

Step 6: Turn to the time slot $(t+1)$ and repeat steps 1 to 5 .

H. Scheduling based on Signal-to-Leakage-Plus-Noise Ratio (SLNR) in LTE-A CoMP Systems

To improve cell-average throughput and cell-edge throughput, LTE-A has adopted the term Coordinated Multi-Point (CoMP) transmission/reception. In CoMP, several distributed Remote Radio Equipments (RREs) are connected are connected to a central controller (eNB) via dedicated backhaul, to mitigate Inter-cell Interference (ICI). Two modes of operation are defined in CoMP; Coordinated Scheduling/Beamforming (CS/CB) and Joint Processing (JP). In the former, data are transmitted to the scheduled User Equipment (UE) from one RRE with scheduling decisions made among all RREs. In the later, data are transmitted to the scheduled user from multiple RRE simultaneously [17]. To provide support for Resource Allocation (RA) in CoMP scheme, the authors in [17] proposed RA mechanism, in which a group of users are scheduled together based on the Signal-to-Leakage-Plus-Noise Ratio (SLNR) metric. Where the Leakage of a UE $k$ is the amount of power leaked from its serving cell to other UEs scheduled in neighboring cells [18], and its metric is given by Equation (22).

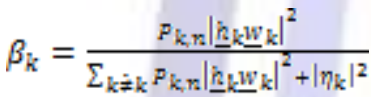

maximize $\beta_{k}$ subject to $\left\|w_{k}\right\|^{2}=1$

Where $\beta_{k}$ is the SLNR of user $k$ on the $n$th PRB, $P_{k_{n} n}$ is the power allocated to the $k$ th UE on the $n$th PRB, $\underline{h}_{k}$ is the complex channel vector of the links between $k$ th UE and all RREs of the CoMP, $\underline{w}_{k}$ is weighting vector that shapes data transmitted from RREs of the CoMP towards $k$ th UE, $\eta_{k}$ is the additive white Gaussian noise at the $k$ th UE. Note that, the numerator of (22) is the signal intended for the $k$ th UE, while the first term of the denominator is the leakage on other UEs from the signal intended to the $k$ th UE.

In the proposed scheme, firstly, the serving $\operatorname{RRE}(s)$ is/are determined. The weighting vector $\underline{w}_{k}$ determines the serving $\operatorname{RRE}(\mathrm{s})$, and then, the users to be scheduled are selected. In CS/CB scheme, since each user is served by only one RRE, all elements of $\underline{w}_{k}$ vector are zero except one element that has unity value and that element represents the serving RRE. While in JP, since each user is served by multiple RREs simultaneously, the elements of the weighting vector (serving RREs) are determined based on Equation (24).

$\underline{w}_{k}=\max \operatorname{eig} . \operatorname{vec}\left(\left(\left|\eta_{k}\right|^{2} I_{M}+\hat{H}_{k}^{*} \hat{H}_{k}\right)^{-1} \underline{h}_{k}^{*} \underline{h}_{k}\right)$

Where $\underline{w}_{k}$ is the eigenvector corresponding to the maximum eigenvalue of the matrix computed in (24), $M$ represents the number of RREs, $I_{M}$ is the $M \times M$ identity matrix and $\hat{H}_{k}$ is a $(K-1) \times M$ matrix given by:

$\hat{H}_{k}=\left[\underline{h}_{1} \underline{h}_{2} \ldots \underline{h}_{k-1} \underline{h}_{k+1} \ldots \underline{h}_{k}\right]^{T}$

Once the serving $\mathrm{RRE}(\mathrm{s})$ is/are determined, a set of users (S) that can share the same PRB are grouped and scheduled together as follows:

Step 1: The user with the maximum SLNR metric is selected to be the first element in the empty set (S).

Step 2: The leakage value from the set $(\mathrm{S})$ is calculated in the direction of the rest of UEs in the CoMP area.

Step 3: The user with the least amount of leakage is selected and placed in the set $\mathrm{S}$; in this manner the least affected UE will share the same PRB with the user belonging to the set $\mathrm{S}$.

Step 4: repeat steps 2 and 3 until the overall leakage value reaches preset value. 
I. Joint Proportional Fairness (JPF) scheduling algorithm for CoMP-SU-MIMO

A scheme for supporting users scheduling in CoMP-Single User-Multiple Input Multiple Output (CoMP-SU-MIMO) mode, where a group of eNBs collaboratively serve only one user over the same PRB, is proposed in [19], and is named Joint Proportional Fairness (JPF) for CoMP SU-MIMO . In the proposed scheme, a cluster of three coordinated eNBs is considered, and users are classified into two groups; cell center UEs and cell edge UEs; based on the received Signal-toInterference-Plus-Noise Ratio (SINR) at each UE. Secondly, priorities of all cell-edge users are calculated based on the CoMP-SU-MIMO mode and the user with the highest priority is selected; and then each eNB of the cluster, using the PF algorithm, selects the user with the highest priority to schedule in non-CoMP-SU-MIMO mode. Thirdly, the following comparison is made.

$3 \times$ prior $_{c_{2} k}<$ prior $_{c_{2} j 1}+$ prior $_{c_{i j} j 2}+$ prior $_{c_{i j} j a}$

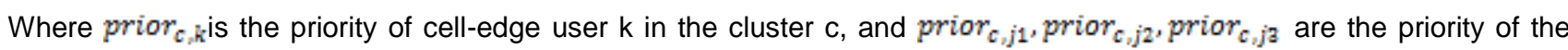
scheduled cell center UE served by eNBs 1,2 and 3, respectively. If the left-hand-side of (26) is greater than the righthand-side, the system will apply CoMP-SU-MIMO on the PRB and cell edge UE is scheduled by coordination among the three eNBs; otherwise, eNBs work on the non-CoMP-SU-MIMO and cell-center UEs are scheduled independently, i.e. without coordination among eNBs.

\section{J. Modified Round Robin (RR) scheduling scheme based CoMP in LTE-A}

To overcome resource conflict and residual resource allocation problems in Joint Transmission (JT) based CoMP (CoMPJT), where multiple eNBs serve single user over the same PRB, the authors of [20] proposed a modified scheduling scheme of Round Robin (RR) algorithm, that works as follows:

Step 1: In each cell users are divided into two groups; cell-edge UEs and cell-center UEs, also, the available frequency bands are divided into two groups; CoMP frequency and non-CoMP frequency.

Step 2: Cell-edge UEs are equally scheduled by their local eNB on PRBs of CoMP frequency zone. The local eNB requests cooperation from neighboring eNBs on the relative PRB. However other eNBs may request cooperation on the same PRB, which is scheduling conflict. In such case, eNBs receiving the cooperation requests follow the rule "first come first served", that is the eNBs respond to the first cooperation request and ignore other requests.

Step 3: Cell-center UEs are equally scheduled in a circular order and are allocated PRBs of single cell frequency band. And if some of cell-edge UEs are not scheduled in the cooperation mode, they will be scheduled as cell-center UEs at the end.

Step 4: Finally, the remaining resources are allocated to cell-center UEs, since they cannot be used for CoMP.

\section{Interference Coordination}

To deal with the growing demand for data services in cellular networks, 3GPP has adopted the term Heterogeneous Network (HetNet) in the LTE-A network. HetNets is a mixed deployment of macro Base Stations (BS) and Low Power Nodes (LPNs), such as Relay Nodes (RNs), picocells and femtocells. The macro BSs are used to provide coverage over wide area, while the LPNs are overlaid onto macro BSs to offload traffic from the macrocells, improving cell-edge performance, and boosting the network capacity. Below we provide a brief description of the different elements of the HetNets [6]:

- Macrocells are operator-installed BSs (in LTE they are called enhanced NodeBs (eNBs)) providing wide area coverage (on the order of few kilometers) and open public access. Typically they emit up to $46 \mathrm{dBm}$ and have dedicated backhaul.

- Picocells are low-power operator deployed cells with similar backhaul and access to the macrocells. Typically they are used in hotspot areas and emit from 23-30 dBm.

- Femtocells, also known as Home eNodeBs (HeNBs), are low-cost low- power cells installed by the end customer. Typically, they are connected to the core network via optical fiber or Digital Subscriber Line (DSL), and operate in open or Close Subscriber Group (CSG) modes and emit less than $23 \mathrm{dBm}$.

- Relays are operator-deployed nodes that are used to improve coverage in poor coverage areas. Typically they emit $30 \mathrm{dBm}$ and backhaul their traffic through a wireless link to a donor eNB.

Inter-Cell Interference $(\mathrm{ICI})$ is one of the key technical challenges that confront the deployment of HetNets, which results from the following reasons [6] [21]:

- Random deployment, femtocells are installed by the end users and without controlling from the network operator, as a result, the conventional network planning becomes inefficient because the network operator does not control the number or location of these nodes.

- $\quad$ CSG-HeNB access, the fact that, access is restricted to a group of subscriber when femtocells work in CSG mode generates significant interference on other users when they are under the coverage area of the femtocells.

- Power difference between nodes, since users are tend to connect to the cell that provides the strongest downlink Received Signal Strength (RSS), the majority of users will be attached to the macro BSs, which leads to the waste of resources in other nodes (RNs and picocells). 
- $\quad$ Range Extension (RE), to overcome the aforementioned problem RE is proposed, in which handover offset value is added to the picocell eNB's Reference Signal Received Signal (RSRP) power, so that UEs are obliged to connect to the LPNs even when they do not provide the strongest RSS. RE has been found useful to mitigate cross-tier interference in the Uplink (UL) direction; however, this comes at the expense of decreasing the Downlink (DL) signal quality of users in the expanded area.

To address the interference problem in LTE-A, where HetNes are adopted, enhanced Inter-Cell Interference Coordination (elCIC) techniques have been exploited in LTE release 10, which are grouped under three main categories according to [6]:

- Time-domain techniques, in which the macro-eNBs reduce their transmission activities in certain subframes, these subframes are known as Almost Blank Subframes (ABS), and the picocells can use these subframes to schedule their users.

- Frequency-domain techniques, the idea behind frequency domain eICIC is to have orthogonal transmission of the physical signals (synchronization and reference signals) at different cells, and that can be achieved by scheduling these signals in a reduced bandwidth.

- Power control techniques, as proposed by 3GPP, ICI can be mitigated by applying different power control schemes at femtocells. If $P_{\max }$ and $P_{\min }$ are the maximum and minimum transmit powers of femtocell, respectively $P_{M}$ is the received power from the aggressor macro-eNB at femtocell, $\alpha$ and $\beta$ are two scalar power control variable, and $P_{t x}$ is transmitted power from the femtocell, then the following power control schemes can be applied at femtocells

a. Strongest macro-eNB received power at femtocell. The transmitted power from femtocell can be written as:

$$
P_{t x}=\max \left(\min \left(\alpha P_{M}+\beta, P_{\max }\right), P_{\min }\right)
$$

b. Path loss between femtocell and Macro-UE (MUE), the transmitted power from femtocell can be written as:

$$
\begin{aligned}
& P_{t x}=\operatorname{med}\left(P_{M}+P_{\text {of st }}, P_{\max } P_{\min }\right) \\
& P_{\text {of } a t}=\operatorname{med}\left(P_{\text {ipl }}, P_{\text {of st }-\max } P_{\text {ofst-min }}\right)
\end{aligned}
$$

Where $P_{\text {ipl }}$ is the indoor path loss and penetration loss between femtocell and MUE, and $P_{\text {off } s t-\max } P_{\text {offst-min }}$ are the maximum and minimum offset values, respectively.

c. Objective SINR of Home-UE (HUE), i.e. attached user to the femtocell, the transmitted power from femtocell can be written as:

$$
\begin{aligned}
& P_{t x}=\max \left(P_{\text {min }}, \min \left(\overline{P L}+P_{\text {red } A U E}, P_{\max }\right)\right) \\
& P_{\text {YEQ AUE }}=10 \log _{10}\left(10^{I / 10}+10^{N_{0} / 10}\right)+\quad S I N R_{\text {tar }}
\end{aligned}
$$

Where $\widehat{P L}$ is the estimated path loss between femtocell and HUE, $I$ is the interference detected by $U E, N_{0}$ is the background noise power and $S I N R_{\text {tar }}$ is target SINR for the HUE.

d. Objective SINR of MUE, the aim of this scheme is to guarantee the minimum SINR of the MUE. And the transmitted power from femtocell can be written as:

$P_{\mathrm{tx}}=\max \left(\min \left(\alpha P_{\text {SINR }}+\beta_{v} P_{\max }\right), P_{\min }\right)$

Where $P_{\text {SINR }}$ is the received SINR power at MUE from the nearest femtocell.

Below we provide some eICIC mechanisms from the literatures:

A. Frequency resources partitioning for Macro-Pico HetNet

In Macro-Pico HetNets, to drive more users selecting picocell as a serving node, a bias value is added to the RSRP of picocell to extend its coverage, and then each user selects its serving node based on the criteria given by Equation (33) [22].

CelliD serving $_{\text {arg }}=\max \left\{R S R P_{i}+\right.$ bias $\left._{i}\right\}$

Where bias $_{i}=0$ for macro eNB and bias $s_{i}>0$ for picocell. With range extension, the number of picocell user will increase, however the user in the extension area will suffer a severe interference from macro eNB, because RSS from eNB is larger than that of picocell node for those users. The authors of [22] adopt frequency partitioning scheme to mitigate the aforementioned problem in which; macrocell is allocated at least $50 \%$ of the available bandwidth, and part of the remaining bandwidth is protected for users in range extension area. The percent of the protected resources can be dynamically changed according to the number of users in picocell and range extension areas and is given by Equation 34.

$\eta=\frac{\text { number of } R \text { RUEs }}{\text { number of PUEs }}$

Where $\eta$ is the percent of protected resources for users in the range extension area, RUEs are the users in the extension area and PUEs are picocell UEs. 
B. Proportional Fair Inter-layer Interference Coordination (PFILIC)

Similar to [22], the authors of [23] developed scheme to mitigate cross-tier interference in macro-pico HetNet, where Cell Range Expansion (CRE) is used, and they named it Proportional Fair Inter-layer Interference Coordination (PFILIC). The PFILIC scheme is based on a combination of both frequency and power coordination together with a set of rules that govern resource allocation, to protect users in the expansion area of the picocells. In the proposed scheme, the available spectrum is divided into two sub-bands; Normal Band (NB) and Platinum Band (PB), also, users are classified into two groups; Cell Center Users (CCUs) and Cell Edge Users (CEUs). For picocell, both subbands are transmitted with the maximum power, and CEUs are scheduled only on PB while CCUs are scheduled on $\mathrm{NB}$, and PB if it is not taken by CEUs. For macrocell, only NB is transmitted with the maximum power while PB has a limited transmitted power, and CCUs are scheduled on both sub-bands, i.e. NB and PB, while CEUs are only scheduled on the PB.

C. Inter-Cell Interference Coordination for CoMP

The authors of [24] proposed ICI coordination scheme for downlink CoMP. In the proposed scheme, according to the attenuation between UE and serving eNB(s), users are classified into Joint Transmission (JT) users and non-JT users. JT users are served by more than one eNB simultaneously; while non-JT users are served only by single eNB with scheduling decisions are taken among several eNBs corresponding to a given coordination cluster (this approach is known Coordinated Beamforming (CB)), which suppress interference within the cluster. Concurrently, $C B$ is exploited by all users, i.e. JT and non-JT, to decrease ICI. The CB vectors are determined based on Signal-toLeakage-plus-Noise-Ratio (SLNR) metric given by (22), where leakage is the interference caused by signal intended for a desired UE on the other simultaneously scheduled UEs in the neighboring cells. Once, UEs are categorized into JT UEs and non-JT UEs, the proposed scheme works in two stages as follows: $i$ ) initial scheduling/ beamforming, in which each cell of the CoMP takes its scheduling decisions independently, and the precoder of each scheduled user is designed based on its SLNR metric ii) iterative scheduling/ beamforming, in which each cell updates its scheduling decisions based on other cells' scheduling decisions, which prevents collision on the same resource.

D. Inter-Cell Interference Coordination (ICIC) scheme based on region

To mitigate the affect of $\mathrm{ICI}$ in HetNets, the authors of [25] proposed ICIC scheme based on regions. In which, cell border is divided into several segments and each is assigned an identity, and the neighboring cells have a full knowledge of all these segments identities. Then, when the interference exceeds a pre-configured value, the serving cell sends to all neighboring cells a combination of the interfered PRB and the region identity in which the UE is located. In turn, each neighboring cell judges if it has caused interference. If so an appropriate ICIC action, such as avoiding the high interfering PRB or reducing power on this PRB, is taken.

E. Interference Coordination for Multi-Hop with relaying

The use of the Relay Nodes (RNs) within cellular network is seen as an effective way to decrease the transmission distance between users and network, and hence boosts the users data rate. However with the universal reuse scheme of one, the co-channel interference among BSs and RNs may increase, and hence the network performance is degraded. In [26], the authors proposed two schemes that can be used to mitigate the effect of the $\mathrm{ICl}$ in multi-hop relaying networks. In the first scheme, ICIC is performed either in the frequency domain or in the time domain, so it is called one-dimensional interference coordination scheme. In this scheme, several RNs are grouped together; they can be grouped for example according to the interference power that $\mathrm{RN}$ can tolerate with acceptable communication, to reuse the same resources. Then in the frequency domain and before allocating radio resources, different priorities of the radio resources for different users are defined. The cell edge users are allocated radio resources with the highest priority, and signal to those users are transmitted with the maximum power. While the cell center user, where interference is limited, are allocated radio resources with the least priority and minimum transmitted power. In the time domain, communication between BS and UE can take place through one of the following links: in the downlink $(\mathrm{BS} \rightarrow \mathrm{RN}$ and then $\mathrm{RN} \rightarrow \mathrm{UE}$ or $\mathrm{BS} \rightarrow \mathrm{UE}$ ), in the uplink direction (UE $\rightarrow \mathrm{RN}$ and then $\mathrm{RN} \rightarrow \mathrm{BS}$ or $\mathrm{UE} \rightarrow \mathrm{BS}$ ). To coordinate the interference between these links, in each sector different links are allocated different subframes, while the same type of links in neighboring cells are not overlapped in the time domain. Additionally, beamforming is used between $\mathrm{BS}$ and $\mathrm{RN}$ to reduce the interference on the link between RN and UE. The second scheme called twodimensional interference coordination (i.e. interference coordination in both time and frequency domains) is used where more than one RN is used per sector. In this situation, intra-sector interference can be dealt with by the frequency domain coordination, while inter-sector interference is dealt with by the time-domain coordination.

F. Cognitive Interference management for Femtocells

The authors of [27] proposed cognitive interference management to coordinate interference among femtocells in LTEA network. In the proposed scheme, neighboring femtocells (HeNBs) exchange information of Component Carriers (CCs) usage and pathloss measurements, via the HeNB gateway or Over The Air (OTA) method, to avoid competing on the same CCs. Firstly, when the femtocell is powered on, it will select the anchor CC (or known as Primary Component Carrier (PCC)). The femtocell will detect whether there is/are neighbor(s) or not. If no, then the femtocell randomly selects one CC as a PCC, else, it has to find which CCs have been used by its neighbors. With the knowledge of that, the HeNB must select CC that is not being used by its neighboring HeNB(s). If all CCs are used by its neighbors, then it selects the one that is used by the furthest and least number of neighbors. If the PCC cannot fulfill the service required by the user, then more CCs must be allocated to that user. At this stage, HeNBs do not exchange pathloss values via the HeNB gateway or by OTA, but the UE measures the pathloss from the neighboring HeNBs and reports it to the serving HeNB, and then the serving HeNB calculates the mutual interference and based on it decides which $\mathrm{CC}(\mathrm{s})$ can be granted. 


\section{Handover Control}

LTE-A network is required to support high-speed mobility terminals; up to $500 \mathrm{~km} / \mathrm{h}$. with this speed, handover will occur more frequently. Therefore, handover decisions have crucial role in determining the overall system performance, especially the real time services. Generally, handover can be defined as the process of changing the access point on the radio link [28]. There are two types of handover in the wireless networks, Hard Handover (HHO) and Soft Handover $(\mathrm{SHO})$. In $\mathrm{HHO}$, also called break-before-make, UE can only communicate with the target eNB after releasing the link with the source eNB. On the opposite side, in SHO, also called make-before-break, UE can communicate with both source and target eNBs during handover process. Currently, LTE and LTE-A systems only support $\mathrm{HHO}$. Compared to the SHO, $\mathrm{HHO}$ is fairly simple and less complex. However, there are several shortcomings of this technique, such as high outage probability, disruption time, carrier interference and data loss, therefore the handover procedure become unreliable, especially for broadband applications and multimedia services [29].

Through surveying the literature, we provide some of the proposed schemes to improve the performance of the handover in the LTE and LTE-A, and these schemes are:

A. Fractional Soft Handover (FSHO) Scheme

Fractional Soft Handover (FSHO) scheme has been developed by the authors of [30] for LTE-A based on Carrier Aggregation (CA) technique. The idea behind FSHO scheme is to partially perform SHO for VolP services. In this scheme, services are classified into VolP services and non-VolP services. When handover is needed, VolP services are transmitted from both source eNB and target eNB, while non-VolP services are either transmitted from source eNB or target eNB (the one with the best signal quality). Theoretical analysis and simulation results in showed that the proposed scheme achieves the lowest handover outage probability and satisfies the QoS of VolP services compared to the both $\mathrm{SHO}$ and $\mathrm{HHO}$ schemes. Additionally it saves radio resources and improves the spectrum efficiency compared with SHO scheme. Finally, the FSHO scheme is backward compatible with LTE system.

B. Combined Handover $(\mathrm{CHO})$ Scheme

Another scheme that utilizes CA for handover in LTE-A is proposed by the authors of [28] and called Combined Handover $(\mathrm{CHO})$ scheme. The objective of this scheme is to reduce the outage probability of handover in LTE-A by physical separation and combined transfer between user data and control information during handover region by $\mathrm{CA}$ technique. This could be achieved by allowing one CC to have access to the target eNB while maintaining packet data through another CCs from the source eNB. It is worth to mention that this scheme is easy to implement and backward compatible with the LTE system.

C. Semi-Soft Handover (SSHO) scheme

A hybrid handover scheme, referred to as Semi-Soft Handover (SSHO) exploiting macro diversity, which allows both $\mathrm{HHO}$ and $\mathrm{SHO}$ advantages for services over multicarrier-based broadband networks to be maintained is proposed by the authors of [31]. The idea of the SSHO is to divide the available bandwidth into two groups; one is for user data and the other is for control data. The control band is then divided into several sub-bands and each eNB in the handover region is allocated one of these sub-bands. The control band comprises the system and control parameters needed for the cell search and handover. During handover, each UE receives multiple control signals from eNBs in the handover region and selects the eNB that has the highest RSRP to receive user data. Due to that, this scheme also called Site Selection Diversity Transmission (SSDT).

D. Combined Partial Reuse and Soft Handover

The authors of [32] proposed an inter-cell interference coordination scheme that relies on a combination of partial reuse and SHO for OFDMA downlink system. The primary objective of this scheme is to improve the cell edge and average cell throughput by considering data rate fairness among all users. The idea of the proposed scheme is to select the better signal quality among the partial reuse scheme and a SHO scheme for the cell edge users. Simulation results in showed that the proposed scheme outperforms the conventional partial reuse scheme in cell edge throughput gain and additionally, it has low soft handover overhead.

E. Joint Processing Handover (JPHO) Algorithm

Joint Processing Handover (JPHO) algorithm was developed by the authors of [33] to support handover in Joint Processing (JP) in CoMP scheme. In this scheme, each UE reports to his serving eNB a set of cells whose RSRP are received by the UE, this set is known as a measurement set. The serving eNB selects from the measurement set the cells with the highest RSRP as CoMP Coordinating Set (CCS). Furthermore, the serving eNB selects from the CCS a group of cells with the highest RSRP as a CoMP transmission Points (CTP). Once the cells in the CTP group start transmission, the UE is required to send measurement update to the serving eNB, so the eNB can search for updated target cells. The handover is triggered when the triggering condition (Equation 35) is fulfilled for the entire Time To Trigger (TTT) time duration followed by the handover command sent from the eNB to the UE.

$R S R P_{T C T P}>R S R P_{S}+H O M$

Where $R S R P_{T} \operatorname{CTP}_{1}, R S R P_{S}$ are the RSRP received by the UE from the target cell in the TCP and serving cell, respectively, and the $\mathrm{HOM}$ is a constant variable that represents the threshold for the difference in the RSRP between the serving cell and the target cell. Once the handover is triggered, the serving cell informs other cells in the CTP to stop transmission to the UE, also instructs the UE to handover to the future-serving cell.

F. Capacity Integrated CoMP Handover ( $\mathrm{CICHO})$ Algorithm

The authors of [5] proposed handover algorithm for CoMP scheme based on the current and historical utilization of the PRBs of the CoMP's cells. The proposed algorithm starts cell selection/reselection once the UE joins the serving cell in the CoMP cluster. Firstly, the serving cell selects the CCS cells (based on Equation 36), and then the cells with 
the highest RSRP in the CCS cells are selected as the CTP cells. The handover will be triggered once the triggering condition (Equation 35) is satisfied for the entire TTT duration otherwise the CTP cells remain transmit to the UE until the next measurement period expires. Measurement period is the time period that is used for checking the handover condition periodically.

capacity $(t) \leq$ capacity threshold

Where capacityc $(t)$ is the capacity indicator of cell $c$ at time $t$, it represents a proportional combination of historical PRB utilize value and PRB utilize value in the current time instant (expressed by Equation 37), and capacity threshold is a constant factor that has a value between 0 and 1 , it is used to select the appropriate target cells whose current and historical capacity able to accommodate the incoming UE.

capacity $_{c}(t)=(1-y) *$ HisRB utilize $_{c}(t-1)+\gamma *$ RB utilize $_{c}(t)$

HisRButilize $_{c}(t)=\frac{\sum_{t=1}^{\mathrm{r}} \text { RButilize }}{\mathrm{c}(\mathrm{t})}$

$R B$ utilize $e_{c}=\frac{\text { REused }_{c}(t)}{R B \max _{c}(t)}$

HisRButilize $_{c}(t)$ is the historical PRB utilize value of cell $c$ from time equal 1 until time $t$, RButilize $e_{c}(t)$ is the PRB utilize value of cell $c$ at time $t$ obtained from Equation $39, R B$ used $d_{c}(t)$ is the total PRBs been used of cell $c$ at time $t, R B \max _{c}(t)$ is the total PRBs of cell $c$ at time $t$ and $\gamma$ is a constant factor that has a value between 0 and 1.

\section{V. conclusion}

This paper has briefly presented some of the technical challenges that face RRM tasks in the LTE-Advanced. It also reviews some of the proposed techniques to solve these issues. PS faces the challenge of supporting diverse QoS requirements of different traffic types, while maintaining the overall system throughput and fairness among users at an acceptable level. Another challenge is the difficulty of scheduling due to the adoption of CA and CoMP scheme. Interference in the LTE-A raises duo the adoption of HetNet. eICIC is the adopted method to relieve its effect which can be grouped under three main techniques: time domain technique, frequency domain technique and power control technique. $\mathrm{HHO}$ is the only handover supported in the LTE and LTE-A. HHO is fairly simple and less-complex compared to the SHO, but $\mathrm{HHO}$ has several disadvantages such as high outage probability, disruption time and carrier interference.

\section{References}

[1] H. Holma and A. Toskala, Lte for umts: evolution to Ite-advanced: Wiley, 2011.

[2] E. Dahlman, S. Parkvall, and J. Skold, 4G: LTE/LTE-Advanced for Mobile Broadband: LTE/LTE-Advanced for Mobile Broadband: Academic Press, 2011.

[3] H. A. M. Ramli, K. Sandrasegaran, and K. Abdullah, "A study of packet scheduling with mobile cellular channel impairments," in Computer and Communication Engineering (ICCCE), 2012 International Conference on, 2012, pp. 888-893.

[4] D. Singhal, M. Kunapareddy, V. Chetlapalli, V. B. James, and N. Akhtar, "LTE-advanced: handover interruption time analysis for IMT-A evaluation," in Signal Processing, Communication, Computing and Networking Technologies (ICSCCN), 2011 International Conference on, 2011, pp. 81-85.

[5] C. C. Lin, K. Sandrasegaran, X. Zhu, and Z. Xu, "On the performance of capacity integrated CoMP handover algorithm in LTE-Advanced," in Communications (APCC), 2012 18th Asia-Pacific Conference on, 2012, pp. 871. 876.

[6] D. Lopez-Perez, I. Guvenc, G. De La Roche, M. Kountouris, T. Q. S. Quek, and J. Zhang, "Enhanced intercell interference coordination challenges in heterogeneous networks," Wireless Communications, IEEE, vol. 18, pp. 22-30, 2011.

[7] S. C. Nguyen, K. Sandrasegaran, and F. M. J. Madani, "Modeling and simulation of packet scheduling in the downlink LTE-advanced," in Communications (APCC), 2011 17th Asia-Pacific Conference on, 2011, pp. 53-57.

[8] R. Kausar, Y. Chen, and K. Chai, "An intelligent scheduling architecture for mixed traffic in LTE-Advanced," in Personal Indoor and Mobile Radio Communications (PIMRC), 2012 IEEE 23rd International Symposium on, 2012, pp. 565-570.

[9] Y. Wang, K. I. Pedersen, T. Sorensen, and P. E. Mogensen, "Utility Maximization in LTE-Advanced Systems with Carrier Aggregation," in Vehicular Technology Conference (VTC Spring), 2011 IEEE 73rd, 2011, pp. 1-5.

[10] Y. L. Chung, L. J. Jang, and Z. Tsai, "An efficient downlink packet scheduling algorithm in LTE-Advanced systems with Carrier Aggregation," in Consumer Communications and Networking Conference (CCNC), 2011 IEEE, 2011, pp. 632-636.

[11] Y. Wang, K. I. Pedersen, T. B. Sørensen, and P. E. Mogensen, "Carrier load balancing and packet scheduling for multi-carrier systems," Wireless Communications, IEEE Transactions on, vol. 9, pp. 1780-1789, 2010.

[12] S. Songsong, F. Chunyan, and G. Caili, "A resource scheduling algorithm based on user grouping for LTEAdvanced system with carrier aggregation," in Computer Network and Multimedia Technology, 2009. CNMT 2009. International Symposium on, 2009, pp. 1-4. 
[13] L. Liu, Y. H. Nam, and J. Zhang, "Proportional fair scheduling for multi-cell multi-user MIMO systems," in Information Sciences and Systems (CISS), 2010 44th Annual Conference on, 2010, pp. 1-6.

[14] Z. Ji-hong, L. Hui, and Q. Hua, "A SPF-PF crossing Component Carrier joint scheduling algorithm," in Advanced Communication Technology (ICACT), 2012 14th International Conference on, 2012, pp. 173-177.

[15] S. Nguyen and K. Sandrasegaran, "Optimised proportional fair algorithm for long-term evolution-advanced system with multiple component carriers," IET Communications, vol. 6, p. 1579, 2012.

[16] L. LIN, Y. LIU, F. LIU, G. XIE, K. LIU, and X. GE, "Resource scheduling in downlink LTE-advanced system with carrier aggregation," The Journal of China Universities of Posts and Telecommunications, vol. 19, pp. 44-123, 2012.

[17] R. A. Abdelaal, M. H. Ismail, and K. Elsayed, "Resource allocation strategies based on the Signal-to-Leakageplus-Noise Ratio in LTE-A CoMP systems," in Wireless Communications and Networking Conference (WCNC), 2012 IEEE, 2012, pp. 1590-1595.

[18] X. LI, Q. CUI, Y. MU, and X. TAO, "Effective resource scheduling scheme considering scheduling relevancy for downlink joint transmission system," The Journal of China Universities of Posts and Telecommunications, vol. 19, pp. 16-25, 2012.

[19] J. Liu, Y. Chang, Q. Pan, X. Zhang, and D. Yang, "A novel transmission scheme and scheduling algorithm for CoMP-SU-MIMO in LTE-A system," in Vehicular Technology Conference (VTC 2010-Spring), 2010 IEEE 71st, 2010, pp. 1-5.

[20] W. Zhou, W. Chen, Z. Tan, S. Chen, and Y. Zhang, "A modified RR scheduling scheme based CoMP in LTE-A system," in Communication Technology and Application (ICCTA 2011), IET International Conference on, 2011, pp. 176-180.

[21] L. Lindbom, R. Love, S. Krishnamurthy, C. Yao, N. Miki, and V. Chandrasekhar, "Enhanced Inter-cell Interference Coordination for Heterogeneous Networks in LTE-Advanced: A Survey," arXiv preprint arXiv:1112.1344, 2011.

[22] C. H. Huang and C. Y. Liao, "An interference management scheme for heterogeneous network with cell range extension," in Network Operations and Management Symposium (APNOMS), 2011 13th Asia-Pacific, 2011, pp. $1-5$.

[23] C. S. Chiu and C. C. Huang, "An Interference Coordination Scheme for Picocell Range Expansion in Heterogeneous Networks," in Vehicular Technology Conference (VTC Spring), 2012 IEEE 75th, 2012, pp. 1-6.

[24] Q. Cui, S. Yang, Y. Xu, X. Tao, and B. Liu, "An Effective Inter-Cell Interference Coordination Scheme for Downlink CoMP in LTE-A Systems," in Vehicular Technology Conference (VTC Fall), 2011 IEEE, 2011, pp. 1-5.

[25] J. Huang, P. Xiao, and X. Jing, "A downlink ICIC method based on region in the LTE-Advanced system," in Personal, Indoor and Mobile Radio Communications Workshops (PIMRC Workshops), 2010 IEEE 21st International Symposium on, 2010, pp. 420-423.

[26] K. Zheng, B. Fan, J. Liu, Y. Lin, and W. Wang, "Interference coordination for OFDM-based multihop LTEadvanced networks," Wireless Communications, IEEE, vol. 18, pp. 54-63, 2011.

[27] L. Zhang, L. Yang, and T. Yang, "Cognitive interference management for Ite-a femtocells with distributed carrier selection," in Vehicular Technology Conference Fall (VTC 2010-Fall), 2010 IEEE 72nd, 2010, pp. 1-5.

[28] Y. Lee, J. Zhao, and H. Qu, "A combined handover scheme for LTE-advanced system," in Advanced Communication Technology (ICACT), 2012 14th International Conference on, 2012, pp. 154-157.

[29] I. Shayea, M. Ismail, and R. Nordin, "Advanced handover techniques in LTE-Advanced system," in Computer and Communication Engineering (ICCCE), 2012 International Conference on, 2012, pp. 74-79.

[30] J. Chang, Y. Li, S. Feng, H. Wang, C. Sun, and P. Zhang, "A fractional soft handover scheme for 3GPP LTEadvanced system," in Communications Workshops, 2009. ICC Workshops 2009. IEEE International Conference on, 2009, pp. 1-5.

[31] H. Lee, H. Son, and S. Lee, "OFDM-Based Semi-Soft Handover for High Data Rate Services," in Personal, Indoor and Mobile Radio Communications, 2007. PIMRC 2007. IEEE 18th International Symposium on, 2007, pp. $1-5$.

[32] C. S. Chiu and C. C. Huang, "Combined partial reuse and soft handover in OFDMA downlink transmission," in Vehicular Technology Conference, 2008. VTC Spring 2008. IEEE, 2008, pp. 1707-1711.

[33] X. Xiaodong, C. Xin, and L. Jingya, "Handover Mechanism in Coordinated Multi-Point Transmission/Reception System," in ZTE Communications, vol. 8, 2010. 